5. EITM. Empirical implications of theoretical models. http://www. nsf.gov/sbe/ses/polisci/reports/pdf/eitmreport.pdf. Accessed October 2, 2012

6. Clarke KA, Primo DM. Overcoming the 'physics envy.' http:// www.nytimes.com/2012/04/01/opinion/sunday/the-social-sciencesphysics-envy.html. Accessed October 2, 2012

7. Windschitl M, Thompson J, Braaten M. Beyond the scientific method: model-based inquiry as a new paradigm of preference for school science investigations. Science Education 2008;92:941-67

8. Musante S. A dynamic alternative to the scientific method: AIBS eye on education. http://www.aibs.org/eye-on-education/eye_on_education_ 2009_01.html. Accessed October 2, 2012

9. Understanding science. How science really works. http://undsci. berkeley.edu. Accessed October 2, 2012

EDITORIAL

\section{Acute Stroke Imaging Research Roadmap II and International Survey of Acute Stroke Imaging Capabilities: We Need Your Help!}

M. Wintermark and S.J. Warach,

on behalf of the STIR and Virtual International Stroke Trials Archive (VISTA)-Imaging Investigators

$\mathbf{P}$ erforming neuroimaging in the setting of a clinical trial, across multiple sites, is challenging because it involves standardizing acquisition and processing imaging protocols on multiple types of scanners by using multiple different platforms. The challenge is even more pronounced for cutting-edge imaging techniques such as arterial spin-labeling or diffusion tensor imaging. Mechanisms are therefore needed to translate and test advanced imaging methods across centers, to encourage the use of advanced imaging in acute settings, to stimulate closer academic-industry collaborations, and to promote the retrospective and prospective collection and pooling of imaging data while keeping in mind practical considerations such as clinical feasibility.

This daunting task has been tackled by the Stroke Imaging Research (STIR) group, a consortium of neuroradiologists, neurologists, imaging scientists, and emergency physicians with an interest in stroke imaging. STIR had a series of meetings in 2012 and 2013, where heated debates led to consensus recommendations as part of a stroke imaging research roadmap. This roadmap was published in Stroke 1 and should be read by all radiologists interested in stroke research because it contains some very important recommendations in terms of standardization of image acquisition and processing for stroke and how imaging should be incorporated in stroke clinical trials. To view the paper use the link in this issue's table of contents, or go directly to: http:// stroke.ahajournals.org/lookup/doi/10.1161/STROKEAHA.113. 002015 .

STIR proposes a specific, standardized terminology for acute stroke imaging, aligned with the National Institute of Neurological Disorders and Stroke Common Data Elements, ${ }^{2}$ including a modified TICI scale to assess reperfusion on cerebral conven- tional angiography. STIR also introduces the concept of "Treatment-Relevant Acute Imaging Targets" (TRAIT), which is meant to capture imaging elements needed for inclusion (or exclusion) into specific treatment protocols. TRAIT acts as a shorthand term to describe the collection of specific imaging metrics used in protocols and simultaneously reminds trial designers to ensure that imaging is directed to the key anatomic or physiologic targets of their specific intervention.

STIR proposes the establishment of a calibration process for measuring ischemic core and penumbral software, as well as the population of the STIR clinical and imaging data repository to facilitate this calibration process. STIR recognizes that imaging techniques continuously evolve and that there will always be a newer, better ischemic core or penumbral imaging technique or processing software. Therefore, it is desirable to find a balance between continued attempts to improve on existing methods versus determining whether existing methods are good enough to be used in current clinical trials. At this time, STIR does not assess or recommend how to use ischemic core and penumbral information for prognosis, prediction of response to treatment, and/or selection of patients for reperfusion therapy. These are better answered in well-designed clinical trials or prospective validation studies.

Finally, STIR recommends the creation of a stroke neuroimaging network involving a collaboration between sites to promote scientific collaboration and education in a distributed fashion and further advance imaging protocols and software reuse, and data and model sharing. As a first step towards the creation of this network, STIR is conducting an international survey for which we need your help. Please take 15 minutes to fill out the survey, which can be found at https://www.surveymonkey.com/s/DQRDYB2. Thank you in advance for your collaboration!

\section{REFERENCES}

1. Wintermark M, Albers GW, Broderick JP, et al. Acute Stroke Imaging Research Roadmap II. Stroke 2013 Jul 16 [EPub ahead of print]

2. http://www.ninds.nih.gov/research/clinical_research/toolkit/ common_data_elements.htm Accessed July 12013

\section{EDITORIAL}

\section{Mechanical Thrombectomy after IMS III, Synthesis, and MR-RESCUE}

\author{
L. Pierot, J. Gralla, C. Cognard, and P. White
}

$\mathbf{T}$ hree recent publications report the neutral results of 3 randomized studies (Synthesis Expansion, Interventional Management of Stroke [IMS] III, and Mechanical Retrieval and Recanalization of Stroke Clots Using Embolectomy [MR-RESCUE]) comparing IV thrombolysis therapy with the endovascular treatment (EVT) of acute ischemic stroke (AIS). ${ }^{1-3}$ The simultaneous publication of these 3 reports might lead to the erroneous conclusion that endovascular treatment has no place in the management of AIS. However, the role of endovascular therapy for the treatment must be more carefully considered, given the tremendous

http://dx.doi.org/10.3174/ajnr.A3654

AJNR Am J Neuroradiol 34:1669-73 Sep 2013 www.ajnr.org 1671 
evolution that imaging and endovascular treatment modalities have undergone over the past several years. Careful analysis of the IMS III, Synthesis, and MR-RESCUE studies shows that these reports have shortcomings as the result of changes in imaging and device technology and study designs.

Over the years, treatment of AIS has evolved to include IV thrombolysis and endovascular treatment. Several randomized studies of IV thrombolysis indicated little to no protection of ischemic brain before it was first reported that IV tPA administered within the first 3 hours after stroke onset had protective effects. ${ }^{4}$

The endovascular treatment of AIS has evolved, moving from intra-arterial (IA) chemical thrombolysis to mechanical thrombectomy. IA chemical thrombolysis was evaluated in several randomized trials that demonstrated its efficacy and safety. ${ }^{5}$ The next advancement in endovascular treatment of AIS was mechanical thrombectomy. Originally, this was performed with no specific tools by the injection of saline within the clot, disruption of the clot with a microguidewire, and "angioplasty" of the clot with remodeling balloons. Subsequently, dedicated, first-generation devices, such as the Merci and Penumbra devices, were developed to catch or aspirate the clot by different means. These first-generation devices were followed by second-generation devices (eg, "stentrievers" such as Solitaire), which were developed to promptly restore blood flow through retrieval of the clot. ${ }^{6}$

Until now, there have been no randomized, controlled trials (RCTs) that compared endovascular treatment with IV tPA for the management of AIS. IMS III, Synthesis, and MR-RESCUE are the first RCTs comparing EVT with IV tPA for the management of AIS and therefore merit attention.

IMS III is an international, phase 3, randomized, open-label clinical trial with a blinded outcome, comparing a combined approach of IV tPA followed by endovascular treatment with standard IV tPA treatment. From August 2006 to April 2012, 656 participants in 58 centers underwent random assignment (approximately 2 patients per center per year). The trial was stopped early because of futility, as defined by the prespecified aim $(10 \%$ difference in Rankin scale score of $\leq 2$ at 90 days). The proportion of patients with good outcome was slightly but not significantly higher in the EVT group (40.8\% in the EVT arm and $38.7 \%$ in the IV tPA arm). There was also no significant difference between the 2 groups in mortality rate at 90 days as well as in the rate of symptomatic intracerebral hemorrhage within 30 hours after initiation of tPA. During the long inclusion period (close to 6 years), imaging and endovascular treatment modalities underwent a tremendous evolution, and utilization of this new technology was only partially implemented in the IMS III protocol, leading to major weaknesses in assessing the data. Only 306 of 656 participants $(46.6 \%)$ had preoperative CTA, and it was not used for inclusion. Clearly, the inclusion of patients without a major arterial occlusion in a randomized trial dealing with endovascular recanalization illustrates an important weakness of the IMS III trial. Moreover, in patients for whom CTA was obtained, the rate of partial or complete recanalization was different in the EVT and IV tPA groups ( $81 \%$ and 35\%, respectively, for an occlusion in the internal carotid artery; $86 \%$ and $68 \%$ for an M1 occlusion; and $88 \%$ and $77 \%$ for an M2 occlusion). Therefore, in terms of recanalization, mechanical thrombectomy performed better than IV
tPA alone for all locations, and, as demonstrated in IMS III, the proportion of patients with good clinical outcome increased with greater reperfusion, indicating that when analyzed this way, mechanical thrombectomy is associated with good clinical outcome. Another important limitation of the IMS III study is that the modalities of endovascular treatment were heterogeneous: IA tPA administration (37.8\% of patients), mechanical thrombectomy with first-generation devices (34.3\%), and only $1.2 \%$ with second-generation devices. As the SWIFT trial has clearly demonstrated, the second-generation devices are more efficacious than first-generation devices in terms of both recanalization and clinical outcome. ${ }^{6}$ Thus, the results of IMS III were outdated before they were published.

Synthesis is an Italian randomized, multicenter clinical trial with a blinded end point, comparing standard IV tPA treatment (initiated within 4.5 hours after symptom onset) to endovascular treatment (within 6 hours after symptom onset). Patients who were assigned to the EVT group did not receive IV tPA. All EVT modalities were authorized. The demonstration of vessel occlusion was not a precondition for inclusion in this trial. From February 2008 to April 2012, 362 patients underwent random assignment (3.5 patients per center per year). The primary end point (disability-free survival at 90 days, mRS 0 or 1 ) was similar in both groups (30.4\% in the endovascular group and $34.8 \%$ in the IV tPA group). Symptomatic intracranial hemorrhage within 7 days occurred in $6 \%$ of patients in both groups. Additionally, death at 90 days was not significantly different between the 2 groups.

Although the inclusion period was shorter in Synthesis than in IMS III (4 versus 6 years), the same important limitations were encountered in Synthesis. Modern CT or MR modalities were not used to visualize vessel occlusion or evaluate penumbra. Additionally, EVT was mostly IA tPA, and second-generation devices were not commonly used (13.9\%). Two other important limitations were encountered in Synthesis. Patients with NIHSS score as low as 2 were included, and they have a very high probability of having a good recovery at 3 months regardless of treatment given. Also, IV tPA was not given in the endovascular group. Synthesis compared IV tPA treatment with isolated endovascular treatment and not with combined IV tPA treatment and mechanical thrombectomy. As a consequence, the endovascular group received treatment 1 hour later than the IV tPA group, which, in part, explains the relative equivalence of endovascular treatment and IV tPA treatment. Given this, it is not surprising that the Synthesis study reconfirms the IMS III finding regarding the limited efficacy of endovascular treatment performed with obsolete tools.

The MR-RESCUE trial was a small, phase $2 \mathrm{~b}$, randomized, controlled, open-label, multicenter trial conducted at 22 sites in North America ( 0.7 patient per center per year). Patients between the ages of 18-85 years with NIHSS scores of 6-29 who had a large-vessel, anterior circulation ischemic stroke were randomly assigned within 8 hours after the onset of symptoms to undergo either mechanical embolectomy (with first-generation devices) or to undergo standard medical care. All patients underwent pretreatment multimodal CT or MR imaging of the brain, which permitted stratification according to the presence of a favorable penumbral pattern versus a nonpenumbral pattern. Among 118 eligible patients (recruited over 7 years), 64 were assigned to the 
embolectomy group and 54 were assigned to the standard care group. In total, 68 of 118 patients (57.6\%) had a favorable penumbral pattern after pretreatment imaging. The 118 patients were classified into 4 groups: embolectomy/penumbral (34 patients); standard care/penumbral (34 patients); embolectomy/nonpenumbral (30 patients); and standard care/nonpenumbral (20 patients). Statistical analysis testing to determine whether there was an interaction between treatment assignment and penumbral pattern determined no significance. Mean 90-day mRS scores were not significantly different between the groups. The number of patients with good outcome at 90 days (mRS 0-2) was also not significantly different between the groups. Death rates also did not significantly differ.

Unlike the IMS III or Synthesis trials, pretreatment evaluation was more precise, with CTA or MRA used to depict large-vessel proximal anterior circulation occlusion, and multi-modal CT or MR imaging of the brain was used to evaluate penumbral status. However, the MR-RESCUE trial still had several drawbacks. The number of patients in each group was small ( $\leq 30$ patients), leading a low statistical power for their analysis. As in Synthesis and IMS III, first-generation thrombectomy devices were used, and it is now clear that efficacy is limited with these devices relative to second-generation thrombectomy devices. Additionally, the management of penumbral patterns is not easy to use in real time. Despite the development of specific models, the study software processed only $58 \%$ of cases successfully in real time. Additionally, the final pattern assignment changed after core laboratory postprocessing in an alarmingly high percentage of cases $(8 \%)$. Also, the use of 2 different imaging modalities (CT and MR) to evaluate penumbra further complicates the findings in this study. It should also be noted that the time to enrollment for all patients in this study, regardless of assigned group, was relatively long (from 5.2-5.8 hours), and this must have played a large role in the overall disappointing clinical outcomes in the trial.

In summary, IMS III, Synthesis, and MR-RESCUE are 3 important trials that perfectly illustrate the difficulty of randomization for techniques that are in rapid evolution.

The main weaknesses of these trials are:

- Long period of inclusion (all studies): Difficulty in the recruitment of patients was encountered in most RCTs dealing with endovascular treatment. Mechanical thrombectomy is not yet a validated treatment, and, by not including all their relevant patients in RCTs, physicians take the risk of having this technique not validated.

- Small number of patients per center per year (all studies).

- Inappropriate preoperative imaging including absence of CTA or MRA to detect an occlusion of a major arterial trunk (IMS III and Synthesis).

- No evaluation of the salvageable brain with perfusion CT or MR (IMS III and Synthesis).

- Comparison of IV tPA to EVT alone (Synthesis and MR-
RESCUE): It does not appear to be the best course of action because the combined approach (endovascular treatment plus IV tPA) allows the physician to start treatment early and to synergize the efficacy of chemical and mechanical thrombolysis.

- Heterogeneity of the endovascular techniques used, with most of them no longer used. IA administration of fibrinolytics, as well as treatment with first-generation thrombectomy devices, has become outdated with the development of second-generation, stent-based thrombectomy devices. The clinical impact of the use of these novel devices has been impressively illustrated by the recent SWIFT trial. ${ }^{6}$

Analysis of the IMS III, Synthesis, and MR-RESCUE studies reveals what steps must be taken next in evaluating the role of endovascular therapy for the treatment of AIS. Analyzing these studies clearly shows that the selection of patients (clinical status, initial extension of ischemic lesion, depiction of arterial occlusion depicted, evaluation of salvageable brain) and the therapeutic methods evaluated are key elements that must be addressed in the design of future studies.

\section{ACKNOWLEDGMENTS}

We thank Nagesh Uppuluri, $\mathrm{PhD}, \mathrm{MBA}$, for editorial assistance in the preparation of the manuscript.

ICMJE Disclosures: Laurent Pierot—RELATED: Consulting fee or honorarium: Codman, Covidien, MicroVention, Penumbra, Sequent. Jan Gralla—RELATED: Consulting fee or honorarium: Global PI of the STAR study, therefore consultant for Covidien*; UNRELATED: Grants/grants pending: Peer-reviewed funding of the Swiss National Fund.* Christophe Cognard_UNRELATED: Consultancy: Codman, Covidien/ev3, MicroVention, Sequent, and Stryker. Philip White-UNRELATED: Consultancy: MicroVention, Terumo; Grants/grants pending: Acandis, Codman, Covidien/ev3, MicroVention,* Terumo, ${ }^{\star}$ Comments: Grant pending for phase II trial. I am also co-PI for PISTE trial, which has some industry support, but that goes to the University of Glasgow, not my institution; Payment for Lectures (including service on speakers bureaus): Covidien/ev3, Codman ( ${ }^{*}$ money paid to institution).

\section{REFERENCES}

1. Ciccone A, Valvassori L, Nichelatti M, et al. Endovascular treatment for acute ischemic stroke. N Engl J Med 2013;368: 904-13

2. Broderick JP, Palesch YY, Demchuk AM, et al. Endovascular treatment after intravenous t-PA versus t-PA alone for stroke. $N \mathrm{Engl}$ J Med 2013;368:893-903

3. Kidwell CS, Jahan R, Gornbein J, et al. A trial of imaging selection and endovascular treatment for ischemic stroke. $N$ Engl J Med 2013;368:914-23

4. The National Institute of Neurological Disorders and Stroke rt-PA Stroke Study Group. Tissue plasminogen activator for acute ischemic stroke. N Engl J Med 1995;333:1581-87

5. Furlan A, Higashida R, Wechsler L, et al. Intraarterial prourokinase for acute ischemic stroke: the PROACT II study: a randomized controlled trial. JAMA 1999;282:2003-11

6. Saver JL, Jahan R, Levy EI, et al. Solitaire flow restoration device versus Merci retriever in patients with acute ischaemic stroke (SWIFT): a randomized, parallel-group, non-inferiority trial. Lancet 2012;380:1241-49 